\title{
Invasive Pulmonary Aspergillosis
}

National Cancer Institute

\section{Source}

National Cancer Institute. Invasive Pulmonary Aspergillosis. NCI Thesaurus. Code C162141.

A form of aspergillosis characterized by the presence of septate, acute, branching hyphae invading lung tissue. 